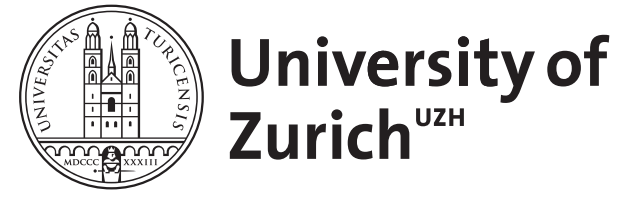
Archive

University of Zurich

University Library

Strickhofstrasse 39

CH-8057 Zurich

www.zora.uzh.ch

Year: 2017

The relevance of anesthetic drug-induced neurotoxicity

Hansen, Tom G ; Engelhardt, Thomas ; Weiss, Markus

DOI: https://doi.org/10.1001/jamapediatrics.2016.3481

Posted at the Zurich Open Repository and Archive, University of Zurich

ZORA URL: https://doi.org/10.5167/uzh-128631

Journal Article

Published Version

Originally published at:

Hansen, Tom G; Engelhardt, Thomas; Weiss, Markus (2017). The relevance of anesthetic drug-induced neurotoxicity. JAMA Pediatrics, 171(1):e163481.

DOI: https://doi.org/10.1001/jamapediatrics.2016.3481 


\section{The Relevance of Anesthetic Drug-Induced Neurotoxicity}

Tom G. Hansen, MD, PhD; Thomas Engelhardt, MD, PhD; Markus Weiss, MD, PhD

Preclinical studies ${ }^{1-3}$ in young animals have demonstrated neurodegeneration and subsequent neurocognitive impairment for virtually all clinically available general anesthetic drugs. However, comparative human studies on this issue are scarce and inconclusive.

Several reasons account for this discrepancy. First, preclinical animal studies were never driven by a clinical suspicion of neurocognitive deficits associated with exposure to anesthetic drugs in early life. There is no welldefined and specific long$\leftarrow$ Related article term phenotype associated with exposure to anesthetic drugs. The first preclinical investigations were extrapolated from findings related to fetal alcohol syndrome and long-term fetal exposure to antiepileptic drugs. ${ }^{3}$ An a priori expectation that anesthetic drugs also would be neurodegenerative in comparable experimental settings was based on the putative (but unknown) mechanisms of actions of anesthetics. ${ }^{3}$ A large number of studies, reviews, and commentaries have since been published as a result. ${ }^{4,5}$

There are many challenges that need to be overcome when translating animal studies into a human context. ${ }^{3,6}$ If exposure to anesthetic drugs was indeed as harmful in early human life as hypothesized, ${ }^{4,5}$ this effect would most likely have been suspected many years ago.

Some observational cohort studies ${ }^{7-9}$ have demonstrated an association between negative long-term neurocognitive outcomes in young children exposed to anesthesia (and surgery), perpetuating any anxiety. Most of these observational investigations are from single centers (prone to selection bias) reporting on small sample sizes with large age ranges (and very few neonates and infants) and a multitude of surgical procedures. Inconsistent outcome measures confuse the public and professionals alike, resulting in a failure to demonstrate a human corollary to this animal phenomenon. However, what constitutes a meaningful human outcome measure? What is important for the child, the parent, and the public interest?

The most commonly reported neurocognitive outcome measures in humans are IQ, learning disabilities, academic performance, neuropsychiatric disorders (autism and attentiondeficit/hyperactivity disorder), and individual neuropsychological test results (eg, Bayley and Wechsler scores). ${ }^{3}$ All of these outcome measures have limitations. A global neurocognitive decline (affecting IQ, learning disabilities, and attention-deficit/ hyperactivity disorder) may be the consequence of an indiscriminate effect of anesthetic drugs. If specific brain areas are damaged during susceptible developmental times, the relevant neurological outcome may be subtle (eg, reading, speak- ing, attention, and fine motor movements). ${ }^{10}$ However, the most important aspects of these outcome measures for a family are neglected. How well does a single short-term interim measure performed in early childhood or adolescence adequately predict outcome and social functioning later in life, and what are the long-term consequences? To what extent are sensorimotor deficits ${ }^{3}$ relevant?

Learning disabilities are ill defined and influenced by many underlying circumstances, such as chronic diseases and environmental factors. They are a nonspecific neuropsychological outcome measure and are categorized according to the predicted and actual educational achievement. Extensive and repeated neurodevelopmental testing is sensitive enough to detect smaller, more minor neurocognitive impairments after anesthesia. ${ }^{11}$ Studies that use individually administered cognitive tests are more likely to detect a potential phenotype (eg, abnormalities in speech and language). ${ }^{9}$ Comprehensive cognitive testing is laborious and expensive. The sample size in most of these studies is small and overestimates the effect size (type I error) or fails to detect a difference (type II error). ${ }^{10,12}$ These concerns are similar to the problem of postoperative cognitive dysfunction in the elderly, with only insufficient tools available to reliably detect its presence. ${ }^{12}$ Therefore, it is essential to ascertain under what circumstances individual cognitive testing represents a meaningful human outcome measure.

Academic performance has a relevant and pragmatic advantage over such testing because of parental interest in how their child performs in school and beyond. More important, are cognitive test results really that different from academic performance? Certainly, good school test scores require adequate speech and learning skills. Good academic achievements are complex and require continuous commitment to the education, social and emotional well-being, and stability of the child.

In this issue of JAMA Pediatrics, a Swedish nationwide cohort study ${ }^{13}$ examining the birth cohorts from 1973 to 1993 reports academic achievement (school grades at age 16 years) and cognitive performance (IQ for boys at military conscription at age 18 years). Exposed children ( $n=33514$ ) who had undergone a single anesthesia and surgery before age 4 years and no subsequent hospitalization were compared with a control group of unexposed children ( $\mathrm{n}=159619)$ matched on sex, maternal educational level, and year and month of birth. The unique sample size of this study enables precise and robust effect estimates.

Glatz et $\mathrm{ll}^{13}$ state that surgical and anesthetic exposure before age 4 years has minimal or no effect on academic achievement or cognitive performance in adolescence. The authors showed that a single exposure or multiple exposures to anesthesia in early childhood are several times (in some cases, 5-10 
times) less relevant to academic performance than sex, maternal educational level, or year and month of birth. These results are similar to those of other robust nationwide cohort investigations. ${ }^{3}$ They are also consistent with the recently published 2-year interim analysis of the General Anesthesia Spinal (GAS) study, ${ }^{14}$ which showed that sevoflurane anesthesia for up to 1 hour in infancy (up to 60 weeks' postmenstrual age) does not increase the risk of adverse neurodevelopmental outcome compared with awake regional anesthesia. These findings are also in line with a recent ambidirectional sibling investigation (the Pediatric Anesthesia Neurodevelopment Assessment [PANDA] study ${ }^{11}$ ) demonstrating similar IQ scores in sibling pairs among whom one was exposed and the other was unexposed to general anesthesia for inguinal hernia repair before age 3 years.

There is an important message to be derived from the study by Glatz et al. ${ }^{13}$ Neonates, infants, and young children need and require important surgical procedures and interventions to be performed without any delay. The authors highlight the need to rectify common ear, nose, and throat problems early and not delay intervention until age 3 or 4 years, thus minimizing the effect of hearing deficits on the development of speech and language skills. This statement is particularly important given that ear, nose, and throat procedures are the most common surgical procedures performed in young children and that most of the supportive observational studies on this topic include a high proportion of children undergoing such procedures.

This study is also reassuring for children, parents, and caregivers and puts the issue of anesthetic-related neurotoxicity and the developing brain into perspective. Glatz et $\mathrm{al}^{13}$ elegantly demonstrated that many other factors are far more important than anesthetic drug exposure in relation to longterm neurocognitive outcomes. However, this finding does not automatically mean that anesthesia is "safe" in young children as this term refers to the conduct of anesthesia rather than the anesthetic drugs used. ${ }^{6,15}$ Also, these results cannot be extrapolated to longer and more complex surgical procedures, multiple anesthetics, and extremely premature infants. Observational studies are plagued by many confounding factors, and randomized clinical trials are complex and time consuming. It will be difficult to achieve another similarly large sample size within a realistic study period.

Ultimately, it seems unlikely that anesthetic drug exposure in young children will be identified as correlating with long-term neurocognitive outcomes. Attention must now be directed to the more important environmental, medical, and individual factors. ${ }^{6}$

\section{ARTICLE INFORMATION}

Author Affiliations: Department of Anesthesiology and Intensive Care-Pediatrics, Odense University Hospital, Odense, Denmark (Hansen); Department of Clinical Research-Anesthesiology, University of Southern Denmark, Odense (Hansen); Department of Anesthesia, Royal Aberdeen Children's Hospital, Aberdeen, Scotland (Engelhardt); Department of Anesthesia, University Children's Hospital, Zurich, Switzerland (Weiss); Faculty of Medicine, University of Zurich, Zurich, Switzerland (Weiss)

Corresponding Author: Tom G. Hansen, MD, PhD, Department of Anesthesiology and Intensive Care-Pediatrics, Odense University Hospital, DK-5000 Odense, Denmark (tomghansen @dadlnet.dk)

Published Online: November 7, 2016. doi:10.1001/jamapediatrics.2016.3481

Conflict of Interest Disclosures: None reported.

\section{REFERENCES}

1. Jevtovic-Todorovic $\mathrm{V}$, Hartman RE, Izumi $Y$, et al. Early exposure to common anesthetic agents causes widespread neurodegeneration in the developing rat brain and persistent learning deficits. J Neurosci. 2003;23(3):876-882.

2. Zhou L, Wang Z, Zhou H, et al. Neonatal exposure to sevoflurane may not cause learning and memory deficits and behavioral abnormality in the childhood of cynomolgus monkeys. Sci Rep. 2015;5:11145.
3. Hansen TG. Anesthesia-related neurotoxicity and the developing animal brain is not a significan problem in children. Paediatr Anaesth. 2015;25(1) 65-72.

4. Rappaport BA, Suresh S, Hertz S, Evers AS, Orser BA. Anesthetic neurotoxicity: clinical implications of animal models. N Engl J Med. 2015;372(9):796-797.

5. Psaty BM, Platt R, Altman RB. Neurotoxicity of generic anesthesia agents in infants and children: an orphan research question in search of a sponsor. JAMA. 2015:313(15):1515-1516.

6. Weiss M, Hansen TG, Engelhardt T. Ensuring safe anaesthesia for neonates, infants and young children: what really matters. Arch Dis Child. 2016; 101(7):650-652

7. Ing C, DiMaggio C, Whitehouse A, et al Long-term differences in language and cognitive function after childhood exposure to anesthesia. Pediatrics. 2012;130(3):e476-e485. doi:10.1542 /peds.2011-3822

8. Wilder RT, Flick RP, Sprung J, et al. Early exposure to anesthesia and learning disabilities in a population-based birth cohort. Anesthesiology. 2009;110(4):796-804.

9. Ing $\mathrm{CH}$, DiMaggio CJ, Malacova $\mathrm{E}$, et al. Comparative analysis of outcome measures used in examining neurodevelopmental effects of early childhood anesthesia exposure. Anesthesiology 2014;120(6):1319-1332

10. Flick RP, Nemergut ME, Christensen K, Hansen TG. Anesthetic-related neurotoxicity in the young and outcome measures: the devil is in the details. Anesthesiology. 2014;120(6):1303-1305.

11. Sun LS, Li G, Miller TL, et al. Association between a single general anesthesia exposure before age 36 months and neurocognitive outcomes in later childhood. JAMA. 2016;315(21): 2312-2320.

12. Lewis MS, Maruff P, Silbert BS, Evered LA, Scott DA. Detection of postoperative cognitive decline after coronary artery bypass graft surgery is affected by the number of neuropsychological tests in the assessment battery. Ann Thorac Surg. 2006; 81(6):2097-2104

13. Glatz P, Sandin RH, Pedersen NL, Bonamy A-K Eriksson LI, Granath F. Association of anesthesia and surgery during childhood with long-term academic performance [published online November 7, 2016]. JAMA Pediatr. doi:10.1001 /jamapediatrics.2016.3470

14. Davidson AJ, Disma N, de Graaff JC, et al; GAS Consortium. Neurodevelopmental outcome at 2 years of age after general anaesthesia and awake-regional anaesthesia in infancy (GAS): an international multicentre, randomised controlled trial. Lancet. 2016;387(10015):239-250.

15. Weiss M, Vutskits L, Hansen TG, Engelhardt T. Safe Anesthesia For Every Tot: the SAFETOTS initiative. Curr Opin Anaesthesiol. 2015;28(3):302307. 